\title{
A Spatial Markov Model for upscaling transport of adsorbing-desorbing solutes
}

\author{
Thomas Sherman ${ }^{\mathrm{a}}$, Amir Paster ${ }^{\mathrm{c}}$, Giovanni Porta ${ }^{\mathrm{b}}$, Diogo Bolster ${ }^{\mathrm{a}}$ \\ ${ }^{a}$ Dept. of Civil and Environmental Engineering and Earth Sciences, University of Notre \\ Dame, IN, USA \\ ${ }^{b}$ Dipartimento Ingegneria Civile ed Ambientale Politecnico di Milano, Piazza L. Da \\ Vinci, 32, 20133 Milano, Italy \\ ${ }^{c}$ BreezoMeter, Haifa, Israel
}

\section{Abstract}

The Spatial Markov Model (SMM) is an upscaled model with a strong track record in predicting upscaled behavior of conservative solute transport across hydrologic systems. Here we propose an SMM that can account for reactive linear adsorption and desorption processes and test it on a simple benchmark problem: flow and transport through an idealized periodic wavy channel. The methodology is built using trajectories that are obtained from a single high resolution random walk simulation of conservative transport across one periodic element. Our approach encodes information about where a particle starts at the inlet, where it leaves at the outlet, how long it takes to cross the domain and one additional piece of information, the number of times a particle strikes the boundary, with the objective of predicting large scale transport with arbitrary linear adsorption and desorption rates. Our benchmark problem demonstrates that predictions made with our proposed SMM agree favorably with results from direct numerical simulations, which resolve the full transport problem.

Keywords: Sorption Desorption, Upscaling, Spatial Markov Model 


\section{Introduction}

Transport of chemical species through porous media can be complex relative to the flow through the medium due to the fact that constituents can sorb and desorb to the solid matrix, thus slowing down their movement relative to the flow. A common textbook approach to account for this is the inclusion of a retardation coefficient in an advection dispersion equation (ADE) [1]. This is only valid when (i) assumptions required to derive the ADE for conservative transport hold and (ii) when one can assume that solute in the fluid and solid phases is in an instantaneous well mixed equilibrium. For complex porous media where geometries can give rise to heterogeneous flows comprised of fast preferential flow channels as well as slower trapping regions, such assumptions can be questionable. Even for conservative transport, where there is no mass exchange between the fluid and the solid phase, anomalous (non-Fickian) transport is known to occur, particularly at early (preasymptotic) times [e.g. 2, 3]. The problem is further complicated with the addition of potentially complex kinetic surface sorption and desorption processes, which introduce their own set of potentially vastly different time scales $[4,5]$.

In many instances, it is not of interest to explicitly describe and resolve all details of transport at all scales, but rather model them effectively at some scale of particular interest. Representing the transport of a solute in a complex flow with a one-dimensional upscaled description can be dated back to upscaling transport in cylindrical tubes by GI Taylor and Aris [6, 7]; this was later generalized to more complex flow configurations using a variety of 
related methods [e.g., 8, 9, 10]. In all cases, longitudinal transport can be described with an effective one dimensional ADE with an enhanced Fickian dispersion coefficient that reflects spreading due to subscale variations in velocities. These models have been generalized to include a wide variety of reactive processes including surface reactions and mixing processes [e.g. 11, 12, 13]. Dykaar \& Kitanidis [12] calculated effective dispersion, velocity and reaction rates in an idealized pore geometry by considering flow and transport in a periodic channel with sinusoidal boundaries where solute can react and degrade close to the boundaries, a process that is mathematically similar to sorption. Levesque et al. [14] generalized Taylor dispersion to systems that include adsorption and desorption to and from solid boundaries in the flow domain and applied it to benchmark Poiseuille flows in planar and cylindrical geometries, both in constant and periodic time varying flows. In all cases effective velocities and dispersion coefficients can vary significantly when compared to values obtained for a conservative solute. These coefficients may also vary dynamically in time as recently shown in [15].

The above methods, in their original form, hinge on the assumption that sufficient time has passed for the solute to sample the full variability of flow velocities under displacements induced by diffusion. This is characterized by the Taylor dispersion time scale $\tau_{D}=L^{2} / D$, where $L$ is a characteristic length scale and $D$ the diffusion coefficient. At times smaller than this, the aforementioned models are strictly speaking not valid. In the presence of reactions, the relative magnitude of reaction and transport characteristic time scales becomes relevant to the applicability of continuum-scale models, such as the standard advection-dispersion-reaction equation [e.g. 16, 17]. Depend- 
ing on the nature of the problem at hand, this may or may not be important. For example, many observations of so called anomalous or non-Fickian transport [e.g. 18, 19, 20] are situations where all length and velocity scales of the system have not yet been sampled and where the largest time scale might be tremendously large relative to times of practical interest [e.g. 21, 22].

It is possible to relax this assumption and develop similar theories that are valid at pre-asymptotic times [e.g. 23, 24, 25], but these still come with strong assumptions that may or may not be met. A strong benefit of these models is that they can help yield great physical insight into important processes at small scales that dominate large scale behaviors. However, in some instances the resulting models can be highly complex integro-differential equations with strong memory effects, meaning that solving them can be as burdensome as solving the full microscale problem [e.g. 26, 25].

The Spatial Markov model (SMM), first introduced by [27] provides an alternative, relatively parsimonious approach that can be applied at preasymptotic times, significantly earlier than Taylor dispersion [28]. The SMM falls into the broad family of continuous time random walk (CTRW) models [29]. In the SMM, a solute plume is represented as a large number of infinitesimal particles that transition through space and time. Most often, spatial increments are fixed and temporal increments are random, sampled from a measured transition time distribution. This is common to many CTRW models. What sets the SMM apart is that successive temporal increments can be explicitly correlated, reflecting underlying persistence of fast particles to move quickly and slow particles to move slowly, which is particularly important in systems that are advection dominated [30]. The SMM has had success 
in upscaling transport across a diverse set of transport settings, including highly heterogeneous Darcy scale porous media [27, 31], fracture networks [32], pore scale systems $[33,34,30,35]$, unsteady flows through porous media [36], and a field scale application to a fractured aquifer [37]. The model has recently been extended to incorporate nonlinear features such as mixing and reactions $[38,39,40]$. In the above examples, the flows are typically either highly heterogeneous with some random structure, or non-uniform, but with a periodic unit cell, as commonly used in classical upscaling approaches such as volume averaging $[9,41]$, the method of moments [8] or homogenization $[10,42]$.

One of the criticisms of the SMM is that it can require extensive parameterization and the most common approach to date is to track Lagrangian particle statistics across two spatial increments, measuring particles transition times across each and representing the correlation structure via a transition matrix. Simplified forms have emerged which assume an idealized structure to the transition matrix and have shown success [37, 43], but the assumed structure may not be sufficiently general to be universal. More recently, an approach was developed that takes successive breakthrough curves and infers the transition matrix structure through an inverse modeling approach [44]. This was later applied to laboratory scale data of transport through zeolite packed columns [45]. All of these approaches require information about travel times across two spatial intervals. Within a domain composed of periodic cells, [40] developed an approach that only requires travel statistics across one cell by parameterizing the model in terms of trajectories rather than just travel times alone. Thus, with one high resolution simulation across 
one cell (corresponding to one spatial increment in the SMM), they were able to efficiently and rapidly predict large scale transport and mixing across much greater extents, with the upscaled model running on the order of 1000 times faster than a fully resolved one.

The work of [40] was only performed in the context of conservative transport. Here we extend this approach to account for (linear) adsorption and desorption processes. In particular, our goal is to only use trajectory statistics from conservative non-sorbing transport across a single periodic cell to predict larger scale transport of a solute that can adsorb and desorb to the solid matrix with arbitrary adsorption and desorption rates; that is, we only need one high resolution simulation across one periodic element with which to ultimately predict a broad and extensive range of possible transport scenarios across large scales. This extension to sorbing solutes opens the pathways towards the application of this methods to a broad class of reactive transport problems, such as contaminant transport in aquifers [46], as well as flow through membranes and packed bed reactors $[47,48]$. In this work we showcase our modeling procedure by relying on a relatively simple flow geometry. The simplicity of the geometry allows for a clear understanding of emergent behaviors which can then be related to observations in more complex settings. Thus, we regard it as an ideal starting point for our current extension of the SMM. Moreover, a simple geometrical setting can provide critical information with which to parameterize pore network models and therefore help bridge the gap between pore- and continuum-scale [e.g. 49]. 


\section{Model system}

\subsection{Pore scale setting}

Figure 1 displays the geometry used in this study, a converging-diverging channel filled with fluid, whose solid boundaries are defined by

$$
h(x)=\bar{h}+h^{\prime} \sin \left(\frac{2 \pi x}{L}-\frac{\pi}{2}\right),
$$

where $x$ is the horizontal coordinate, $h(x), \bar{h}$ and $h^{\prime}$ are related to the width of the half-aperture (see Figure 1), and $L$ is the length of a single cell. In this study, to be consistent with previous ones [e.g. 40, 38], we will focus on the specific values of $\bar{h}=L / 4$ and $h^{\prime}=0.8 \bar{h}$. This setting was first used by [12] to upscale effective reaction rates in porous media using the method of moments. While this geometry is very simple, the emergent flow displays some of the complex features pertinent to understanding flow and transport in porous media, including a fast preferential flow path and stagnant trapping areas, which are known to strongly impact transport in real porous media [e.g. 50]. This, and very similar geometries, have received extensive attention in the literature. For example, $[51,52]$ and $[53,30]$ studied the effects of inertia on flow and transport respectively. Similarly, others have looked at how geometry impacts asymptotic $[54,55]$ and pre-asymptotic transport $[56,57]$, as well as the role of turbulence on large scale dispersion [23]. One of the reasons we choose this geometry is that under the assumption of Stokes flow, i.e. Reynolds number less than $\mathrm{O}(1)$, a semi-analytical solution exists $[58,12]$. The solution uses a perturbation method to solve the biharmonic equation, which governs the streamline structure. Details of the solution, as 


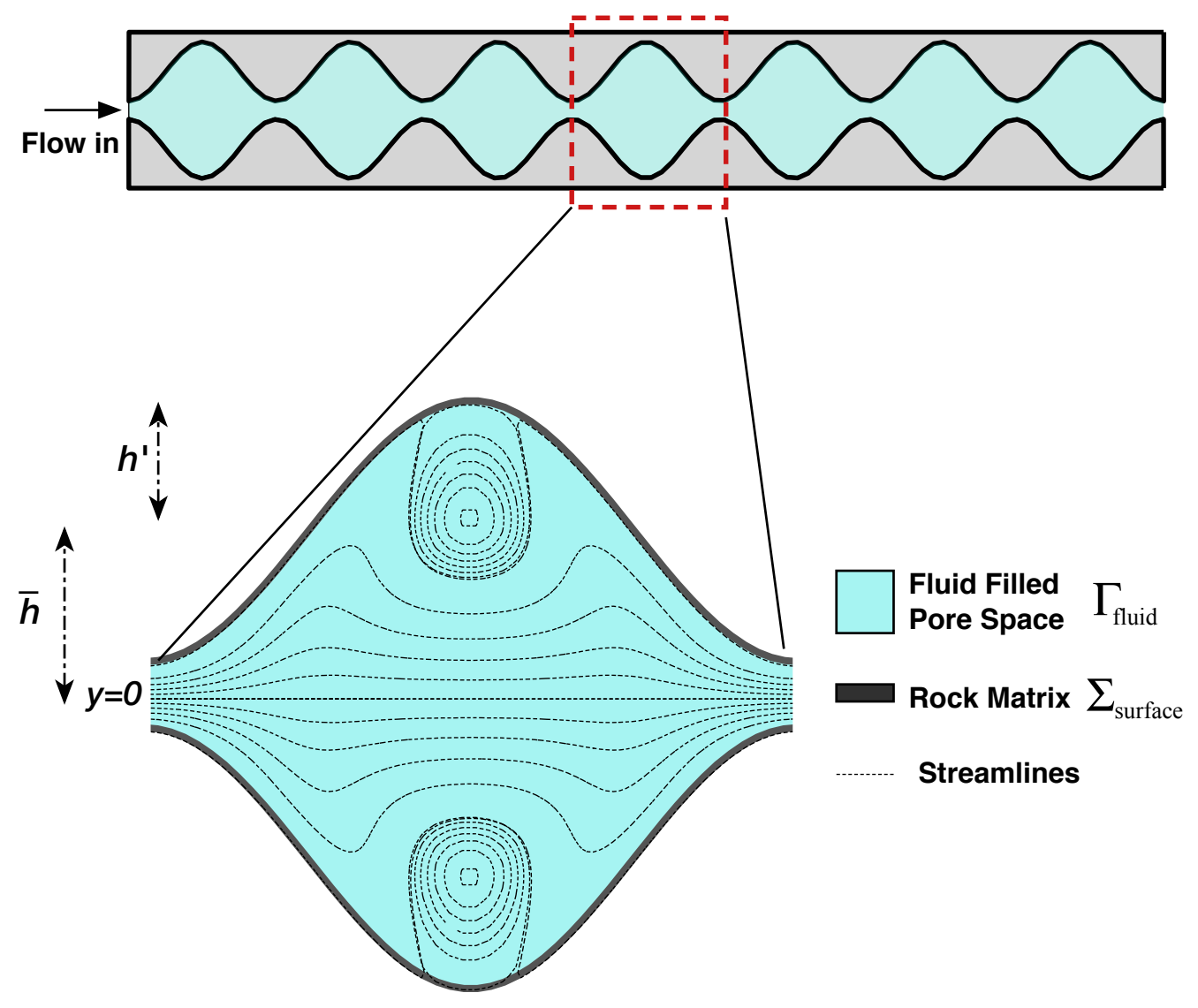

Figure 1: A schematic of the flow domain and the unit pore cell used in this study. 
well as its validation are available in various papers $[58,12,59,55]$ and so are not elaborated on here. The geometry defined above is chosen because of the interesting flow patterns, including the emergence of a recirculation region and a fast central preferential flow channel. While the geometry is highly idealized, these specific features mimic features of interest in real porous media, making this model potentially appealing. Due to the natural symmetry of the system throughout this entire work we only ever simulate the top half of the domain $(y>0)$.

\subsection{Simulation of transport at the microscale}

Solute transport with sorption and desorption is modeled by [60, 15]

$$
\begin{gathered}
\frac{\partial C(\mathbf{x}, t)}{\partial t}+\nabla \cdot[\mathbf{u}(\mathbf{x}) C(\mathbf{x}, t)]=\nabla \cdot[D \boldsymbol{\nabla} C(\mathbf{x}, t)] \quad \forall \mathbf{x} \in \Gamma_{\text {fluid }} \\
\frac{\partial S(\mathbf{x}, t)}{\partial t}=-\lambda S(\mathbf{x}, t)+\alpha C(\mathbf{x}, t)=D \frac{\partial C(\mathbf{x}, t)}{\partial n} \quad \forall \mathbf{x} \in \Sigma_{\text {surface }}
\end{gathered}
$$

where $C(\mathbf{x}, t)\left[M L^{-3}\right]$ is the concentration of the solute in the fluid, $\mathbf{u}(\mathbf{x})$ is the velocity in the fluid phase, $D\left[L^{2} T^{-1}\right]$ is the molecular diffusion coefficient, taken to be constant in the fluid, $S(\mathbf{x}, t)\left[M L^{-2}\right]$ is the concentration on the surface, $\lambda\left[T^{-1}\right]$ is the rate of desorption, $\alpha\left[L T^{-1}\right]$ is the rate of adsorption and $n$ is the unit normal to the boundary. Note that the first equation in (2) describes transport in the main channel, which is governed by the advection diffusion equation. The second equation is the boundary condition, describing exchange between the fluid and solid phase: the rate of change of surface concentration is given by the rate at which solute concentration attaches to the boundary $(\alpha C)$ minus the rate which solid phase concentration is detaching $(\lambda S)$; for mass balance reasons this must be equal 
to the diffusive flux of solute concentration at the boundary $\left(D \frac{\partial C(\mathbf{x}, t)}{\partial n}\right)$. In all cases we consider an initial condition of an instantaneous line source, flux weighted along the pore throat, i.e. $C(\mathbf{x}, t=0) \propto \mathbf{u}(\mathbf{x}) \delta(x)$. This choice of initial condition is common as it is believed to mimic real experiments [e.g. $61,62]$ and also represents the asymptotic distribution to which Lagrangian particles are expected to converge [e.g. 63, 64, 65]

To solve this system we implement a numerical Lagrangian particle based random walk method [66], where the solute plume is discretized into a finite number of $N$ particles. We incorporate the sorption-desorption boundaries following the work of [67]. During each step each particle is moved according to Langevin equation

$$
\begin{aligned}
x_{i}^{n+1} & =x_{i}^{n}+u_{i} \Delta t+\xi_{i} \sqrt{2 D \Delta t} \\
y_{i}^{n+1} & =y_{i}^{n}+v_{i} \Delta t+\eta_{i} \sqrt{2 D \Delta t} \quad i=1, \ldots, N, \\
t_{i}^{n+1} & =t_{i}^{n}+\Delta t+\tau_{i}
\end{aligned}
$$

where $x_{i}^{n}$ and $y_{i}^{n}$ are the horizontal and vertical position of particle $i$ respectively at simulation step $n, u_{i}$ and $v_{i}$ are the $x$ and $y$ components of the velocity respectively, $\xi$ and $\eta$ are independent and identically distributed (iid) Gaussian variables with zero mean and unit variance, $\Delta t$ is a fixed time step, $t_{i}^{n}$ is the time for particle $i$ at simulation step $n$, and $\tau_{i}$ is a random waiting time

$$
\tau_{i}=\left\{\begin{array}{l}
T_{i}: P>U_{i} \\
0: P \leq U_{i}
\end{array},\right.
$$



230

where the $T_{i}$ are iid exponential, with density $\psi(\tau)=\lambda \exp [-\lambda \tau], P$ is the probabilty of sorption and $U_{i}$ are iid $U(0,1)$ (uniformly distributed between 0 and 1). The solid boundaries in the domain are modeled as elastic reflection boundaries. Any time a particle reflects off a solid boundary it either sorbs with probability $P$, resulting in selecting $\tau$ randomly from $\psi(\tau)$, or does not sorb, resulting in $\tau=0$. To leading order, this probability can be computed as

$$
P=\alpha \sqrt{\frac{\pi \Delta t}{D}} .
$$

For further details on this as well as higher order approximations please see [67]. This number $P$ is compared to a random number $U$, drawn from a standard uniform distribution. If $U \geq P$ no sorption occurs and if $U<P$ the particle sorbs.

Our choice of solving this system using this Lagrangian random walk method is based on the following: (i) it naturally aligns with building an SMM, which relies on Lagrangian statistics, (ii) for a periodic system like this one, it is possible to simulate very extensive domains as a particle's velocity at any given time depends only on its local position relative to the periodic cell, meaning that we do not need a prohibitively large Eulerian mesh and (iii) for a sufficiently smooth velocity field, such as this one, it is known not to suffer from numerical dispersion, which could be problematic since diffusion and adsorption processes are closely linked. For all of the results that we present in this paper we used one million particles and a time step of $\Delta t=10^{-3}$, consistent with previous experience in the same domain $[55]$. 


\subsection{Dimensionless numbers}

The system described in section 2.2 is characterized by the following dimensionless numbers

$$
R e=\frac{\bar{h} \bar{u}}{\nu} \quad P e=\frac{\bar{h} \bar{u}}{D} \quad D a_{a}=\frac{\bar{h} \alpha \sqrt{\pi}}{D} \quad D a_{d}=\frac{\bar{h}^{2} \lambda}{D} .
$$

$\bar{u}$ is the mean velocity and $\nu$ is the viscosity of the fluid. Re is the Reynolds number, which we have already assumed to be small $R e<O(1)$ in using the prescribed flow field; $P e$ is the Péclet number, which reflects the competition between advection and diffusion processes and typically lies in the range $0.1<P e<10^{3}[12]$. We will focus on the higher range of these values, as it is known that advection-dominated systems are more likely to violate assumptions inherent to Taylor dispersion and classical advection-disperison upscaling. $D a_{a}$ and $D a_{d}$ are adsorptive and desorptive Damköhler numbers, which respectively compare the time scales associated with adsorptive and desorptive processes to diffusive ones. In a batch system, large values of these would correspond to systems close to equilibrium between surface and fluid concentrations. In the following any reported dimensionless parameters are obtained by setting $\bar{u}=1,2 \bar{h}=1$ (in arbitrary units) and tuning $D, \alpha$ and $\lambda$ to obtain specified values of $P e, D a_{a}$ and $D a_{d}$.

2.4. Inputs for Spatial Markov Model - trajectories, travel times and number of hits

Here we define the inputs that must be obtained from the microscale domain in order to build the macroscale effective SMM, described in the following section. In all cases we obtain these inputs by running a random 
walk with a flux weighted pulse initial condition at the throat of a pore (see figure 1) and simulate transport across one periodic unit cell. This calibration simulation is for a conservative random walk that does not include sorption. The periodic unit cell corresponds to the blown up region in figure 1. Sund et al's [40] SMM is different from others in that it samples trajectories rather than travel times. These trajectories are obtained by simulating transport across a single unit cell, using

$$
\begin{aligned}
& x_{i}^{n+1}=x_{i}^{n}+u_{i} \Delta t+\xi_{i} \sqrt{2 D \Delta t} \\
& y_{i}^{n+1}=y_{i}^{n}+v_{i} \Delta t+\eta_{i} \sqrt{2 D \Delta t}
\end{aligned} \quad i=1, \ldots, N .
$$

This is a standard random walk framework (note these equations are the same as (3), but without the random waiting times associated with modeling sorption). Using this we store specific information about each particle's trajectory. In Sund's approach each trajectory is defined by three parameters: (i) its vertical position at the inlet of the periodic element $y_{i n}$, (ii) the time, $T$, it takes to travel from the inlet to the outlet and (iii) its vertical position at the outlet when it leaves the periodic element $y_{\text {out }}$. In addition to these three inputs, our method will account for adsorption-desorption by storing one additional piece of information (iv) $N_{\text {hits }}$, the number of times a particle strikes a solid boundary when crossing a single cell. We propose that by running one high resolution simulation of conservative transport over one periodic element, we can obtain all of the required information needed to upscale and describe transport with adsorption and desorption efficiently over much larger scales. However, it must be noted that for each different 
value of $P e$, a different calibration simulation is required.

\subsection{Effective transport model - The Spatial Markov Model}

Here we propose an SMM, which is an effective upscaled transport model that describes transport in one dimension aligned with the direction of flow. The SMM is a time domain random walk model and again the solute is represented by discrete particles, whose motion in time and space is governed by:

$$
\begin{aligned}
& \tilde{x}_{i}^{k+1}=\tilde{x}_{i}^{k}+L \\
& \tilde{t}_{i}^{k+1}=\tilde{t}_{i}^{k}+T_{i}^{k}+\sum_{j=1}^{N_{h i t s}^{k}} \tau_{j} .
\end{aligned}
$$

Tildes refer to upscaled quantities. $\tilde{x}_{i}^{k}$ is the horizontal location of particle $i$ at the beginning of step $k, \tilde{t}_{i}^{k}$ is the time associated with particle $i$ at the beginning of step $k, N_{\text {hits }}^{k}$ is the number of times the sampled trajectory strikes the boundary during step $k$. During each step a particle transitions a constant longitudinal distance $L$, the length of our periodic cell, and it does so in a random time $T$, which is sampled from a measured travel time distribution, the discrete distribution obtained from the single pore calibration simulation in (7). $T$ reflects the range of velocities that particles sample in traversing the unit cell. The feature that makes the SMM unique relative to other random walk models is that successive temporal increments are not independent of one another. Correlation arises when a particle that traverses one periodic unit quickly is also likely to traverse the next one 
quickly and likewise for a slow one. In the absence of diffusion, particles persist on the same streamline always and so successive jumps would have the same transition time. Due to diffusion, particles can leave streamlines and sample various flow streamlines. However, when advective effects are strong relative to diffusive ones, memory effects persist and must be accounted for through correlation. In most applications, correlations are applied using a transition matrix [27]. We apply here instead the trajectory based approach recently proposed in [40], because the trajectory based method can account for the number of times each trajectory interacts with the boundary in a way that the original transition matrix approach cannot. This approach naturally provides a framework that more readily characterizes interactions of particles with the reactive boundary, using the information stored as inputs for the SMM described in $\S 2.4$. It should be noted that the periodic nature of the domain is important in accomplishing this, although some other recent studies suggest that trajectory based methods may also work for heterogeneous systems characterized by a stationary heterogeneity distribution $[68,69]$.

In this implementation of the model, each step of length $L$ is associated with a specific particle trajectory. We summarize our algorithm in the following steps:

1. Each particle starts with a given $y_{\text {in }}$ reflecting the desired initial condition. This sets a specific trajectory that has an associated travel time, which is used as the time increment $T_{i}^{k}$ in (8), and an assigned number of hits $N_{\text {hits }}^{k}$.

2. Adsorption is accounted for by the term $\sum_{j=1}^{N_{h i t s}^{k}} \tau_{j}$, where each strike with the boundary adds a possible waiting time, which is sampled from 
the same distribution $\psi(\tau)$ as used in (3) with probability $P$ from (5).

3. The model then samples the next trajectory randomly, using $y_{\text {out }}$ as a conditioning criterion for picking the next trajectory by making sure that its $y_{\text {in }}$ is close to the previous $y_{\text {out }}$. This is done by discretizing the inlet into $N_{\text {bins }}$ equi-sized bins (we used cases with $N_{\text {bins }}=10$, 100 and 1000 in this study with no notable difference in results). A particle's vertical $y$ location determines which bin it is in. Thus $y_{\text {out }}^{k}$ determines the bin from which the next trajectory is sampled. A trajectory with $y_{i n}^{k+1}$ is randomly and uniformly sampled from the same bin that $y_{\text {out }}^{k}$ ends in. This binning procedure ensures correlation effects are accurately imposed.

\subsection{Observables to test model}

We will test the proposed SMM by comparing its ability to predict downstream transport as measured by breakthrough curves (BTCs) measured at multiple downstream locations. These are $x=5 L, 10 L, 25 L$ and $50 L$. Testing the upscaled model against BTCs at multiple downstream locations provides a more robust test of the model compared with focusing on only one location; sometimes a model can match a single BTC due to overparameterization, but when the same model can consistently match observations over multiple scales, it suggests that the underlying physics is being more faithfully captured. Thus, to produce benchmark data against which to test the SMM, we run a series of high resolution random walk simulations using the fully resolved transport equations (3) that account for advection, diffusion, adsorption and desorption. In all cases we use a flux weighted pulse initial condition and run single realization simulations with $N=10^{6}$ particles. 

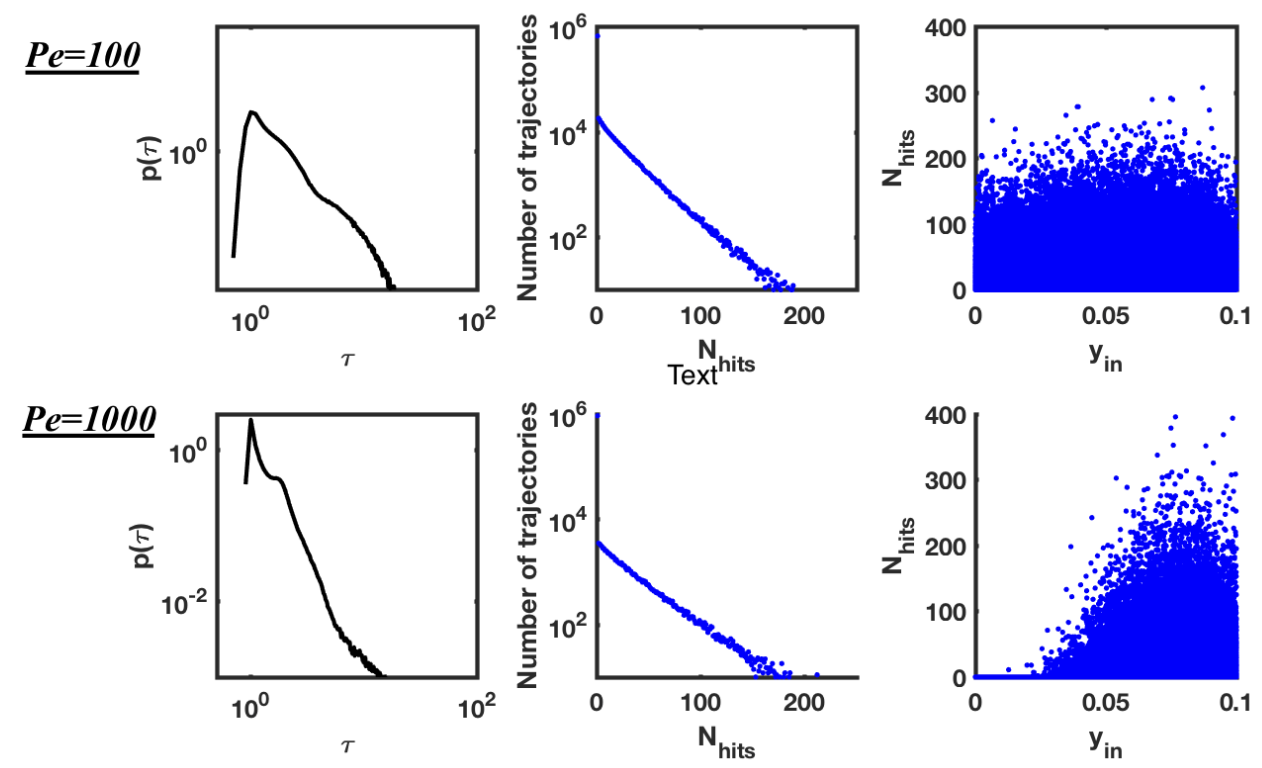

Figure 2: (Left) Travel Times Distributions across single periodic elements (Middle); Frequency scatter plot of number of times each trajectory hit the boundary; (Right) Scatter plot for each simulated particle's starting $y_{i n}$ vs the number of times a particle hits the boundary during one travel time. The top row shows results for $P e=100$ and the bottom row for $P e=1000$.

These results are the reference solution against which the upscaled model is tested.

\section{Results}

\subsection{Travel Time Distributions and Number of Hits}

Figure 2 displays the empirical travel time distributions, measured from simulations, across a single periodic element for two cases, $P e=100$ and $P e=1000$. The data used to plot these travel time distributions is from where temporal increments are sampled. These two Péclet numbers are chosen because, in the case of purely conservative transport, it has been shown 

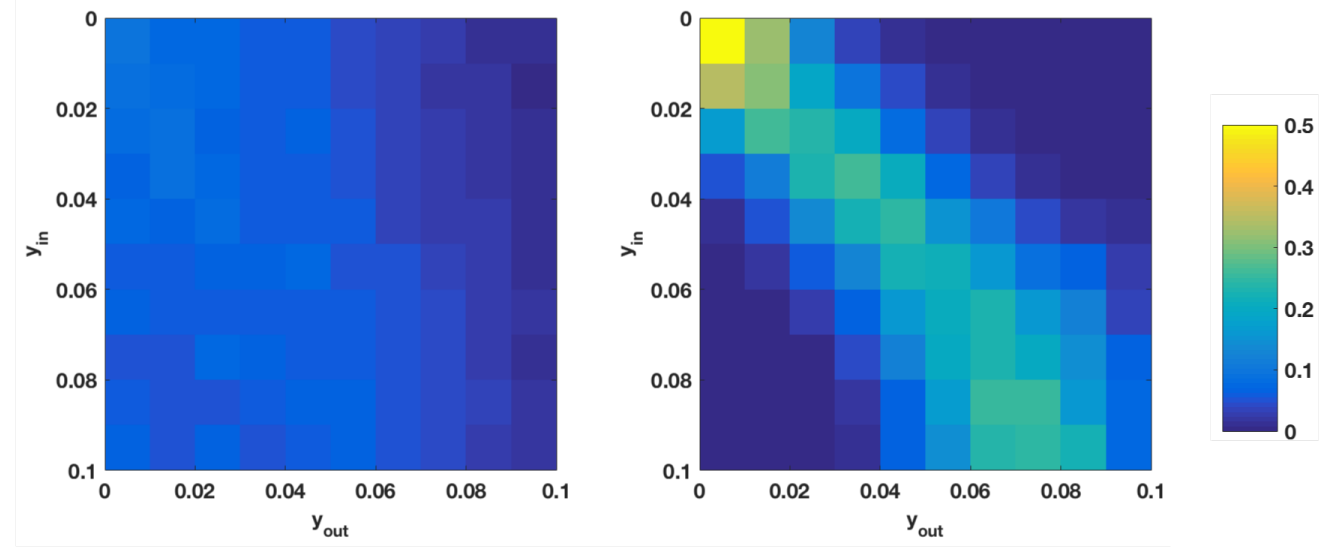

Figure 3: Transition probabilities of $y_{\text {out }}$ given $y_{\text {in }}$ for $P e=100$ (left) and $P e=1000$ (right). In this figure $y_{\text {in }}$ and $y_{\text {out }}$ are discretized into 10 bins of equal size.

that for $P e=100$ incorporating correlation effects is unimportant, while for $P e=1000$ it is [30]. These distributions were obtained by creating a histogram of arrival times and normalizing. The bin size of the histogram grew logarithmically with larger $\tau$.

Also shown are the number of times a particle (trajectory) strikes the boundary during a given transition across a single cell. Most notably, for both Péclet numbers the vast majority (approximately 99\%) of trajectories never actually strike the boundary and pass through the pore with no possibility of adsorption taking place. However, some trajectories can strike the boundary anywhere up to 200 times, meaning that the likelihood of adsorption can be significant depending on the adsorption rate, or the probability of attachment $P$. For the lower Péclet number case more trajectories strike the boundary than in the $P e=1000$ case, which intuitively makes sense given that the surface reaction is diffusion-driven. The expected delay for a reactive particle that strikes the boundary $N_{\text {hits }}$ times is $\langle\tau\rangle=\frac{N_{\text {hits }} P}{\lambda}$, where 
the angled brackets denote the expected value.

Finally, figure 2 shows a scatter plot of each simulated trajectory's starting location at the inlet against the number of interactions with the boundary. This figure clearly highlights that for the $P e=1000$ case a particle has to start near the boundary in order to have any chance of interacting with it. A particle that starts on the centerline $y=0$ has nearly zero likelihood of interacting with the boundary and thus adsorbing. For the $P e=100$ the number of hits per trajectory is relatively independent of $y_{i n}$. This suggests that accounting for correlation effects (i.e. knowing a particle's starting location as it transitions through each pore) may be less important for $P e=100$ than for $P e=1000$, as found in the conservative case [30].

Figure 3 displays the discrete transition probabilities that a particle has for a $y_{\text {out }}$ given a particular $y_{\text {in }}$, which is a measure of correlation. Note that this figure is an approximation of the copula density function defining correlation between travel times in consecutive steps [70]. As has been seen in previous studies for $P e=100$, this matrix is relatively uniform, while there is a stronger diagonal dominance for the $P e=1000$ case reflecting the fact that correlation effects become stronger as Péclet number increases [30].

\subsection{Comparison between DNS and SMM predicted breakthrough curves}

\subsubsection{Limit of no sorption $(P=0)$}

To begin, we demonstrate the proposed SMM's ability to upscale transport in the absence of any sorption at all. Plots comparing breakthrough curves at multiple downstream locations measured by DNS and predicted with the SMM are shown in Figure 4. As in previous studies, the agreement is excellent demonstrating the veracity of our proposed approach. 

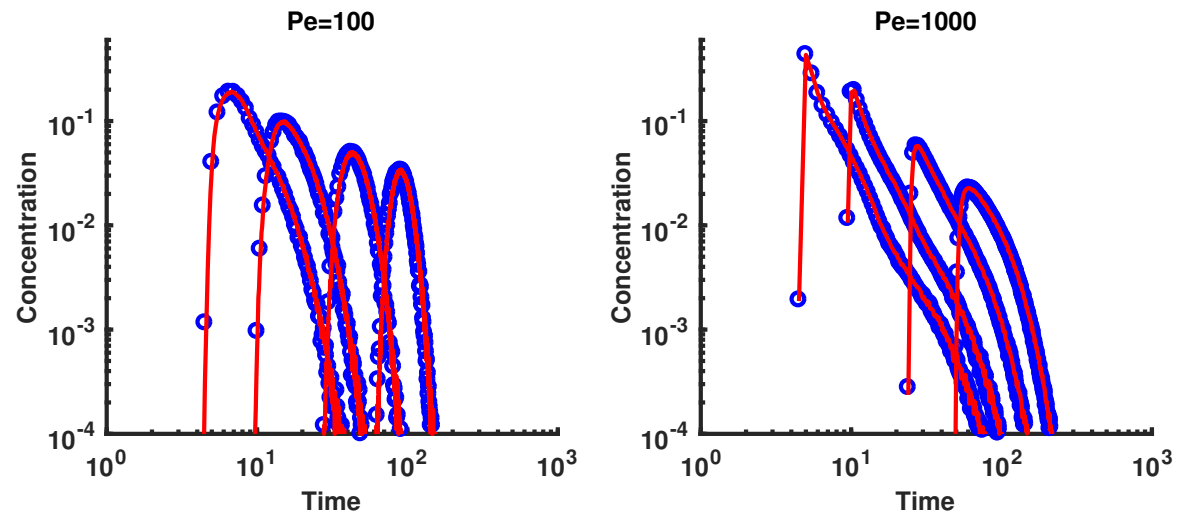

Figure 4: Breakthrough Curves for $P e=100$ (left) and $P e=1000$ (right) at distances of $5 L, 10 L, 25 L$ and $50 L$ with no sorption at all. Blue dots are DNS results and black lines are SMM predictions.

\subsection{Limit of unit probability}

Next we focus on another limiting case, where the probability of sorption each time a particle strikes the boundary is unity $(P=1)$, meaning that every strike results in an adsorption event. While this may not be an entirely physical condition, by considering this extreme case we are testing our proposed procedure across a wide range of possible $D a_{a}$. For $P e=100$ and $P e=1000$ the respective values are $D a_{a}=316$ and $D a_{a}=10^{3}$. The considered values of $\lambda=0.1$ and 1 correspond to $D a_{d}=10,10^{2}$ and $D a_{d}=10^{2}, 10^{3}$ respectively.

Results comparing DNS measured breakthrough curves as well as SMM predicted ones are shown in figure 5. Additionally, for context and to explicitly demonstrate the role of sorption, breakthrough curves for the case of zero sorption, as discussed in the previous section, are also included. Again, the agreement between DNS and SMM is excellent, with the SMM capturing all essential features displayed by the fully resolved simulations. 
For the $P e=100$ case the rising limb of the two earlier breakthrough curves is similar for the cases with and without sorption. This corresponds to fast moving particles following trajectories that never interact with the boundary. The late time behavior and the further downstream breakthrough curves are very distinct with a strong separation between the case with and without sorption, reflecting strong delays due to sorption. For the $P e=1000$ case more particles persist at moving quickly and do not interact with the boundary; thus the early arrivals are similar between cases with and without sorption. Again, at late times there is a deviation between the cases with strong delays in the tails emerging for the cases with sorption.

\subsection{Intermediate sorption}

Results corresponding to a sorbing probability that is one order of magnitude smaller than in the previous section, $P=0.1$, are shown in figure 6 , which correspond to $D a_{a}=31.6$ and 100. The results demonstrate that the SMM provides reliable results across the parameter space.

The resulting breakthrough curves reflect very similar behavior to the previous case; however the tails are not as delayed, reflecting the fact that only $10 \%$ of particles that sorbed in the $P=1$ case actually do so here. For the $P e=1000$ and $D a_{d}=1000$ case $(\lambda=1$, Figure 6 top right), it is visually next to impossible to see differences at late times, while for the longer waiting time $D a_{d}=100(\lambda=0.1$, Figure 6 bottom right $)$ a more distinct delayed tailing behavior emerges for the cases with sorption. Note that breakthrough curves obtained with $P=0.1, \lambda=0.1$ (bottom row in figure 5) are identical to the ones for $P=1, \lambda=1$ case (top row in figure 4 ) because their expected delay times are equivalent. This is because, on average, multiplying both $P$ 

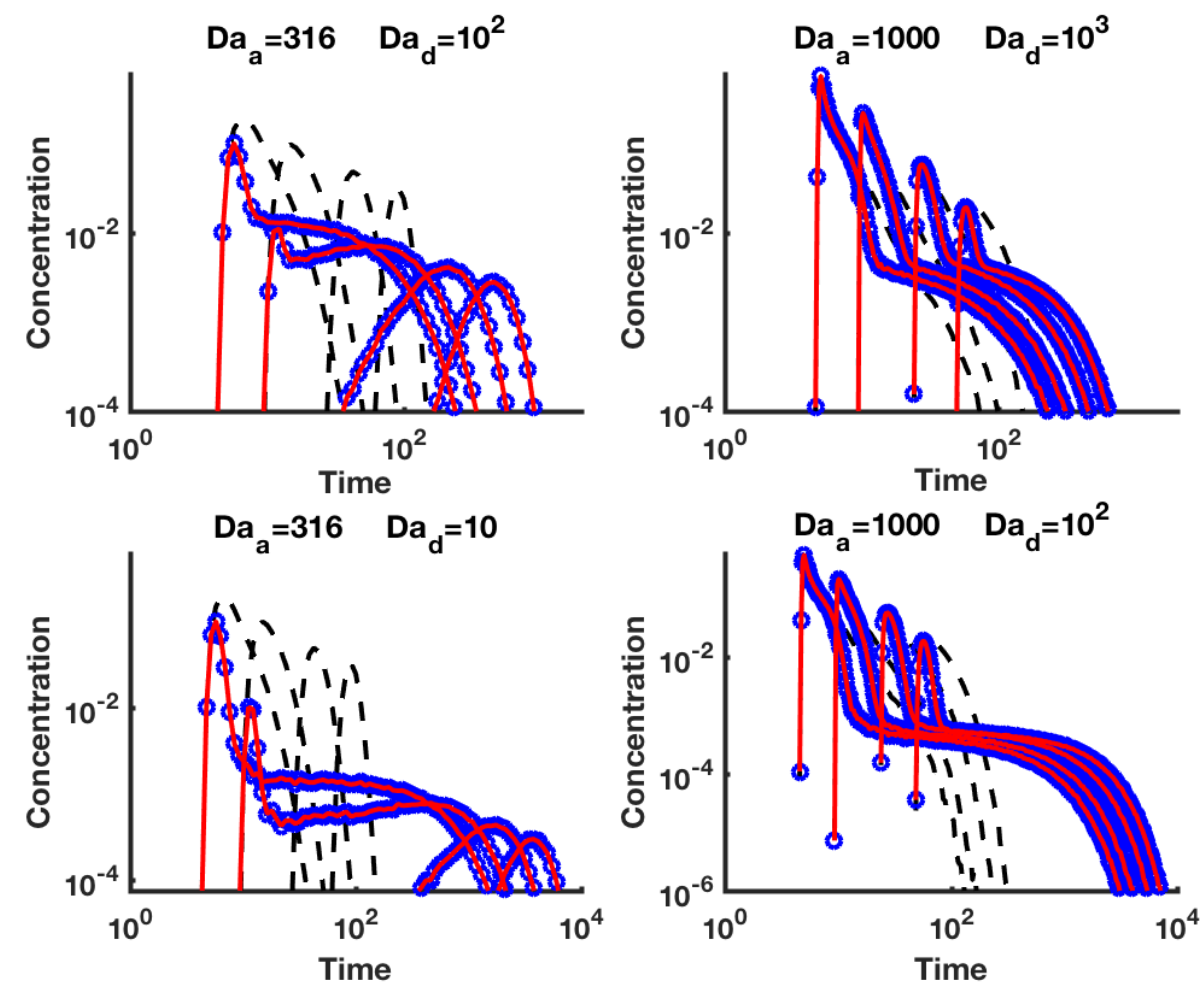

Figure 5: Breakthrough Curves for $P e=100$ (left) and $P e=1000$ (right) at distances of $5 L, 10 L, 25 L$ and $50 L$ with sorption given by $P=1$. Blue dots are DNS results and red lines are SMM predictions. Black dash-dot lines are BTCs for the case without sorption. 



Figure 6: Breakthrough Curves for $P e=100$ (left) and $P e=1000$ (right) at distances of $5 L, 10 L, 25 L$ and $50 L$ with sorption given by $P=0.1$. Blue dots are DNS results and red lines are SMM predictions. Black dash-dot lines are BTCs for the case without sorption.

and $\lambda$ by a constant has no effect on average travel times.

\subsection{The role of correlation}

In this section, we test how important including correlation in the SMM is for accurately predicting downstream reactive transport behavior. To do so, an uncorrelated one-dimensional time domain random walk (TDRW) model is built by simply sampling random trajectories without considering the inlet and outlet locations, which are the quantities by which we enforce correlation in our model. The trajectories are sampled uniformly from the calibration 
simulation data.

Figure 7 shows the comparisons for all parameter choices considered so far, but focusing only the furthest downstream BTC $(x=50 L)$. As expected, the importance of correlation effects is stronger for the $P e=1000$ cases than for $P e=100$. However, even for $P e=100$ distinguishable errors are visible where successive travel times are sampled independently. This suggests that, while correlation effects are seemingly unimportant for the $P e=100$ case for conservative transport [30], as sorption at the boundary occurs, correlation begins to play some role, similar to what has been observed for reactions [38].

Notably, when correlation is not accounted for, predicted breakthrough curves fail to capture the full rising limbs and also display greater delays than the actual measured ones. This reflects the fact that too many particles are interacting with the boundary. The uncorrelated model cannot account for the fact that fast particles have a tendency to persist at staying fast, as well as the fact that the fastest particles have virtually no probability of getting sorbed, as shown in figure 2.

While well known that correlation plays an ever more important role as the Péclet number of a system increases, the effect of boundary reactions on the importance of correlations between successive jumps has not been previously explored. Figure 8 quantifies this effect based on the approximation of particle arrival times. More specifically, we consider the recovered mass at time $t$

$$
M(t)=\int_{0}^{t} C\left(x=50 L, t^{\prime}\right) d t^{\prime}
$$

which is the cumulative distribution function of solute arrival times at $x=$ $50 L$. We compute the mean absolute error $\left(M A E=\sum_{i=1}^{N_{t}} \mid M_{D N S}\left(t_{i}\right)-\right.$ 
$\left.M_{\text {simulated }}\left(t_{i}\right) \mid\right)$ between the results obtained via DNS and the upscaled simulation with uncorrelated steps at location $50 \mathrm{~L}$. Our results, in agreement with previous studies, show that the uncorrelated model can yield accurate arrival times of a nonreactive solute $\left(D a_{a}=0\right)$ for $P e=100$ as the MAE attains a value of $\sim 5 \times 10^{-5}$. The occurrence of adsorption induces an increase of the MAE by approximately two orders of magnitude, irrespective of the probability with which reaction occurs. This is likely because only slower particles ever interact with the boundary and, while weak, some correlation does occur. This does not mean that the model is not sensitive to $P$; indeed it must be since larger values of $P$ result in larger delays. It is only saying that correlation effects may be more important to include in the upscaled model when sorption occurs. Note that when correlation is included the MAE is approximately constant for all adsorption probabilities (including the conservative case, $P=0$ ) and attains a value of approximately $10^{-5}$ (not shown).

Additionally, to provide another basis for comparison, Table 1 shows the arrival times when 1\%, 50\% and $99 \%$ of the mass has arrived for all of the cases shown in Figure 7. We show the dimensionless time for DNS and the relative difference $R_{\Delta}$ between DNS and the two models, computed as

$$
R_{\Delta}(M O D)=\frac{t_{p c}(M O D)-t_{p c}(D N S)}{t_{p c}(D N S)}
$$

where $M O D$ stands for UNC (uncorrelated TDRW) or SMM and $t_{p c}$ indicates the time corresponding to arrivals of percentile $p c$ of total mass (i.e., pc equals $1 \%, 50 \%$ and $99 \%$ here). These results clearly and quantitatively highlight the good agreement between the DNS and SMM as well as the afore- 

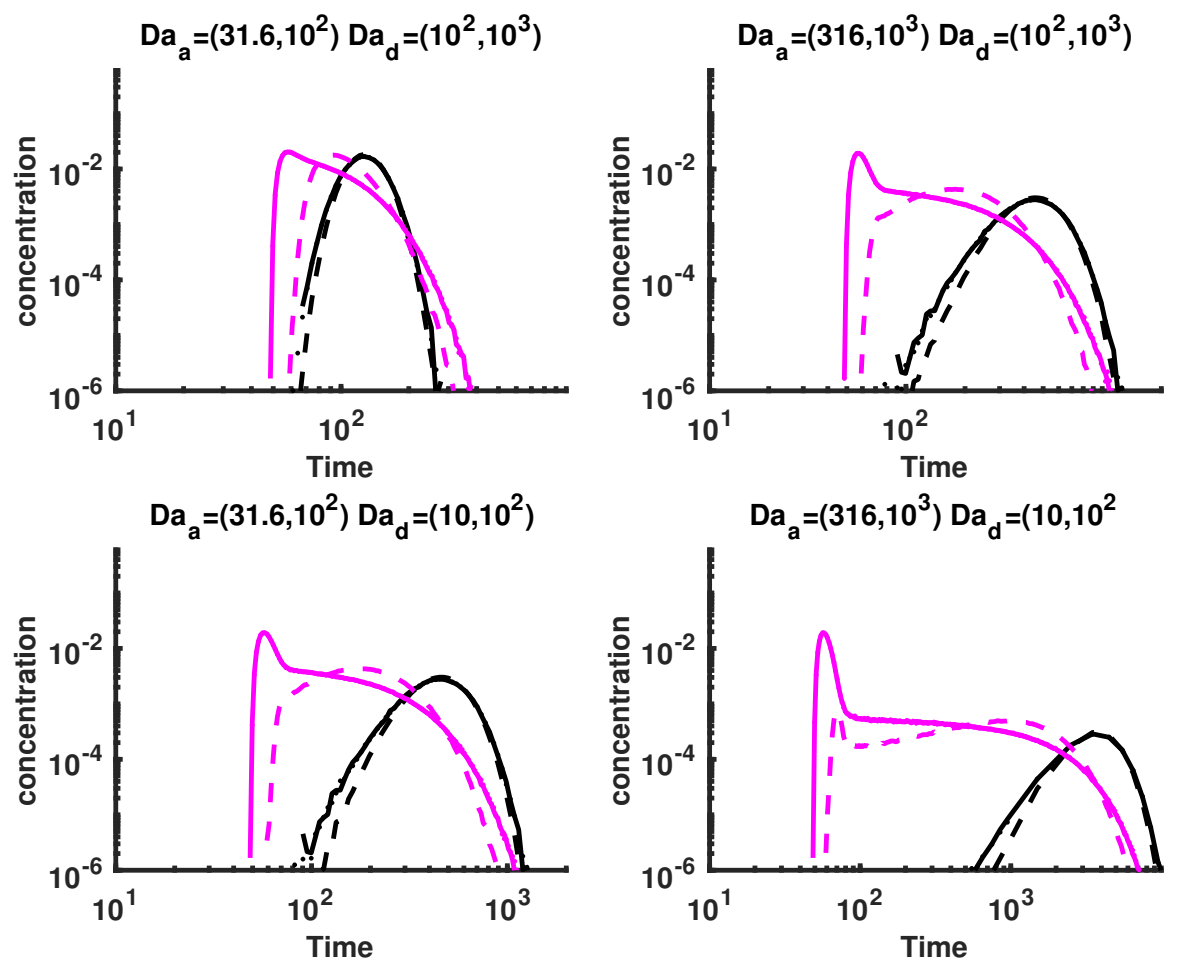

Figure 7: Breakthrough curves at a distance of $50 L$ for $P e=100$ (black) and $P e=1000$ (magenta). Solid lines are the SMM with correlation effects included, while dashed lines do not include correlation effects. Dots are results from the DNS simulations (note that these are hard to see as they coincide so closely with the SMM results). Damköhler numbers in parentheses are for $P e=100$ and 1000 respectively. 


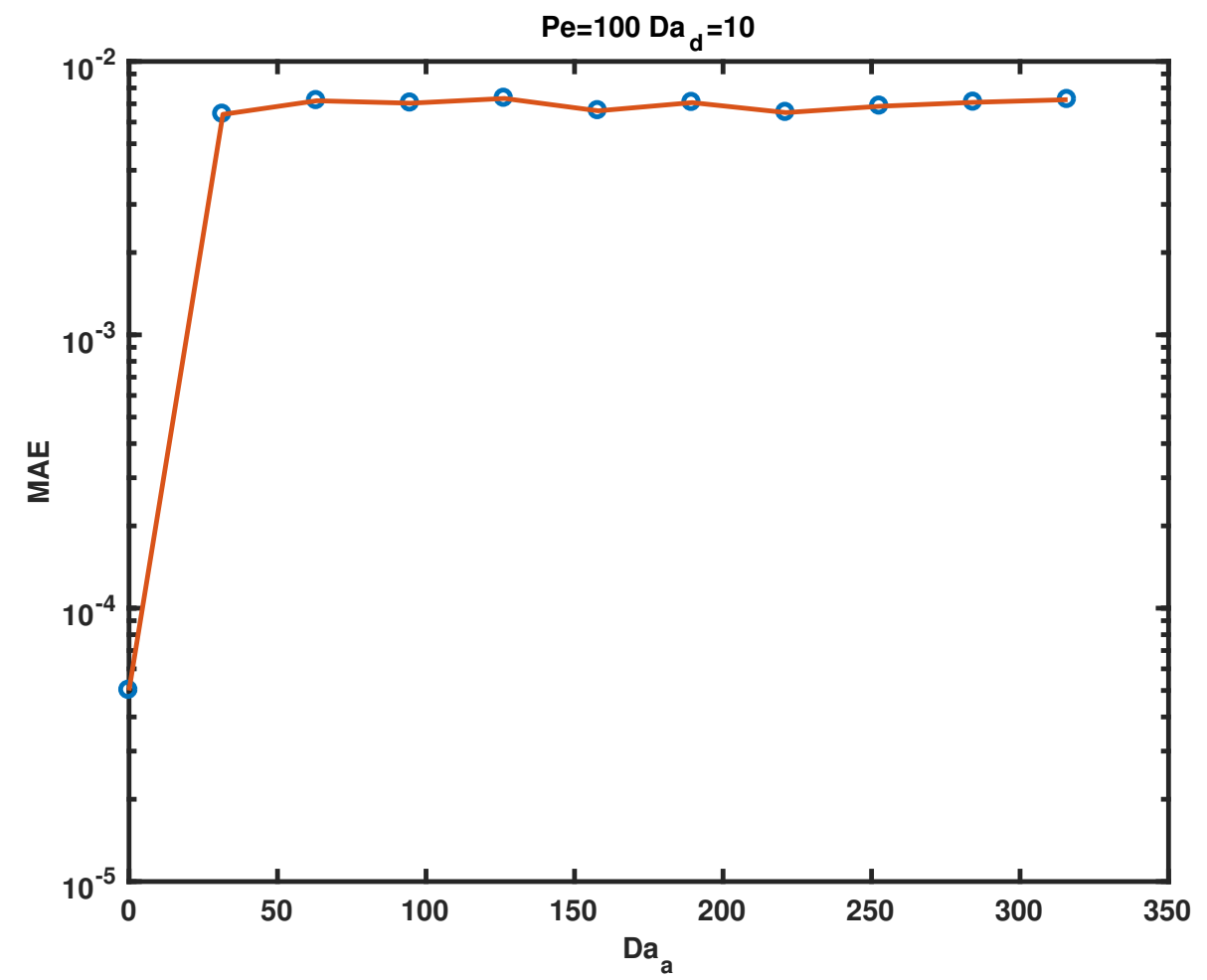

Figure 8: Mean absolute error (MAE) between recovered mass simulated by direct numerical simulation and uncorrelated simulations at $50 L$, for $P e=100$ and $D a_{d}=10$ as a function of $D a_{a}$. 
mentioned discrepancies between the DNS and uncorrelated TDRW model. Differences between DNS and SMM are always within 1\%, showing the robustness of the method. On the other hand, when correlations are neglected, the errors increase sharply with $P e$, i.e. relative differences between uncorrelated model and DNS are up to $10-15 \%$ for $P e=100$ and up to $90 \%$ for $P e=1000$. In general, the uncorrelated TDRW overestimates early arrivals and underestimates late arrivals. Correlation effects also appear to have different influence depending on $D a_{a}$ and $D a_{d}$. These differences emerge in the $1 \%$ and $50 \%$ arrival times (early arrivals and median time), although with relatively smaller variations than the ones observed for $P e$. For early arrivals and median time, the uncorrelated model errors tend to increase for increasing $D a_{a}$ and decrease with increasing $D a_{d}$, i.e. the error may change up to a factor 2 when the two Damköhler numbers change by one order of magnitude.

Table 1: Arrival times for 1, 50, and $99 \%$ of the solute plume to cross $50 L$ in the DNS and relative difference $R_{\Delta}$ related to the upscaled SMM, and uncorrelated TRDW (UNC). Cases correspond to different combinations of adsorptive and desorptive Damköhler numbers.

\begin{tabular}{|c|c|c|c|c|c|c|c|c|c|}
\hline \multicolumn{10}{|c|}{$P e=100$} \\
\hline \multirow[t]{2}{*}{ Case } & \multicolumn{3}{|c|}{$1 \%$ Arrival Time } & \multicolumn{3}{|c|}{$50 \%$ Arrival Time } & \multicolumn{3}{|c|}{$99 \%$ Arrival time } \\
\hline & DNS & $R_{\Delta}(S M M)$ & $R_{\Delta}(U N C)$ & DNS & $R_{\Delta}(S M M)$ & $R_{\Delta}(U N C)$ & DNS & $R_{\Delta}(S M M)$ & $R_{\Delta}(U N C)$ \\
\hline$D a_{a}=31.6, D a_{d}=10$ & 208.5 & $0.58 \%$ & $11.68 \%$ & 478.5 & $0.31 \%$ & $0.71 \%$ & 888.4 & $-0.14 \%$ & $-3.82 \%$ \\
\hline$D a_{a}=31.6, D a_{d}=100$ & 85.9 & $0.23 \%$ & $5.34 \%$ & 130.9 & $0.15 \%$ & $0.46 \%$ & 198.9 & $-0.30 \%$ & $-2.72 \%$ \\
\hline$D a_{a}=316, D a_{d}=10$ & 1416.8 & $0.77 \%$ & $15.09 \%$ & 3953.1 & $0.40 \%$ & $0.73 \%$ & 7800.7 & $0.21 \%$ & $0.73 \%$ \\
\hline$D a_{a}=316, D a_{d}=100$ & 208.6 & $0.48 \%$ & $11.78 \%$ & 478.7 & $0.21 \%$ & $0.73 \%$ & 886.6 & $0.00 \%$ & $-3.46 \%$ \\
\hline
\end{tabular}

\begin{tabular}{|c|ccc|ccc|ccc|}
\hline \multicolumn{10}{|c}{$P e=1000$} \\
\hline$a_{a}=100, D a_{d}=100$ & 52.3 & $0.19 \%$ & $42.94 \%$ & 139.8 & $0.50 \%$ & $52.46 \%$ & 635.1 & $0.60 \%$ & $-13.35 \%$ \\
\hline$D a_{a}=100, D a_{d}=1000$ & 52.3 & $0.19 \%$ & $34.73 \%$ & 84.4 & $0.36 \%$ & $23,26 \%$ & 219.7 & $0.59 \%$ & $-10.18 \%$ \\
\hline$D a_{a}=1000, D a_{d}=100$ & 52.3 & $0.00 \%$ & $76.77 \%$ & 675.2 & $1.13 \%$ & $89.84 \%$ & 4911.7 & $1.09 \%$ & $-12.92 \%$ \\
\hline$D a_{a}=1000, D a_{d}=1000$ & 52.3 & $0.00 \%$ & $42.83 \%$ & 139.9 & $0.43 \%$ & $52.60 \%$ & 635.1 & $0.60 \%$ & $-13.35 \%$ \\
\hline
\end{tabular}




\section{Discussion and conclusions}

We have extended the Spatial Markov Model for periodic flow domains proposed in [40] to account for linear adsorption to and desorption from solid boundaries. In particular, we have built the framework based on a trajectory based SMM, where high resolution trajectories simulated by an advectivediffusive random walk over a single periodic flow element are stitched together sequentially to predict transport over much larger scales. In this novel approach we merely store one additional piece of information about each trajectory, that is the number of times that it strikes a solid boundary. This information reflects the solute flux close to the boundary surface that corresponds to the adsorption reaction rate. Coupling this with a probabilistic representation of sorption [67], we can effectively upscale transport to represent arbitrary adsorption and desorption rates. Thus from a single high resolution random walk simulation of conservative transport across one periodic element we can model an extremely diverse range of adsorption/desorption behaviors without the need to run further high resolution, computationally intensive, small scale simulations for each desired case. Of course this current application is strictly restricted to the example of an idealized periodic setting and it remains to be shown how generalizable it is to more complex and realistic settings.

As in previous studies of conservative transport, the need for the upscaled model to account for correlation between successive jumps depends on the Péclet number of the system, with correlation being more important as advection begins to dominate more and more. Similarly, it appears that the Damköhler numbers play a role in determining this, meaning that merely 
delineating regions where correlation is important or unimportant for conservative transport does not provide a sufficient condition for the case when reaction occurs. This is particularly relevant for the accurate representation of early and late particle arrivals with the upscaled model, because these are most sensitive to correlation effects (fast particles tend to persist as fast and slow as slow). For the conditions explored, we have numerically verified that the correlation between subsequent travel times plays a relevant role across the full range of investigated adsorption rates. As expected, the relevance of correlation is sharply influenced by $P e$, but also increases with $D a_{a}$ (fast adsorption) and decreases with $D a_{d}$ (fast desorption). This trend is particularly striking for early arrivals, which could be of practial relevance, e.g., in assessment of membranes life-cycle or of risk with contaminant breakthrough in aquifers. Notably, our approach conserves the same accuracy with respect to fully resolved simulations for both conservative and reactive transport.

Our model can accurately upscale kinetic sorption and desorption processes, i.e. it does not assume equilibrium between sorbed and dissolved solute mass, as would be the case if modeling adsorption/desorption with a retardation coefficient. We emphasize that the traditional use of a retardation factor to account for the delays in transport induced by adsorption and desorption will not work to reproduce the cases that we simulate here. The main effect of a retardation coefficient would simply be a rescaling in time of a conservative concentration profile or breakthrough curve. However, the breakthrough curves obtained in this study, in many cases, have a fundamentally different shape from those without adsorption and desorption. The use of a retardation coefficient assumes an instantaneous equilibrium 
between mobile and immobile parts of the domain and clearly that is not the case here, particularly for the higher Péclet number case. The persistence of correlation effects is very much inline with the fact that highly mobile particles traveling on fast trajectories have virtually no interaction with the boundaries while slower ones can have many, reflecting a lack of equilibrium. As with upscaling of other transport processes, at late times as systems begin to homogenize (i.e. greater than Taylor time scales) conditions for such an equilibrium can arise, but this may or may not be useful depending on the scales that one is interested in.

\section{Acknowledgments}

This material is based upon work supported by, or in part by, the US Army Research Office under Contract/Grant number W911NF-18-1-0338. The authors were also supported by the National Science Foundation under awards EAR-1351623 and EAR-1417264, EAR-1446236, and CBET-1705770. 
[1] J. Bear, Dynamics of fluids in porous media, Courier Corporation, 2013.

[2] J. Salles, J.-F. Thovert, R. Delannay, L. Prevors, J.-L. Auriault, P. Adler, Taylor dispersion in porous media. determination of the dispersion tensor, Physics of Fluids A 5 (10) (1992) 2348-2376.

[3] D. Lester, G. Metcalfe, M. Trefry, Anomalous transport and chaotic advection in homogeneous porous media, Physical Review E 90 (6) (2014) 063012.

[4] M. Maghrebi, I. Jankovic, R. M. Allen-King, A. J. Rabideau, I. Kalinovich, G. S. Weissmann, Impacts of transport mechanisms and plume history on tailing of sorbing plumes in heterogeneous porous formations, Advances in water resources 73 (2014) 123-133.

[5] M. Maghrebi, I. Jankovic, G. S. Weissmann, L. S. Matott, R. M. AllenKing, A. J. Rabideau, Contaminant tailing in highly heterogeneous porous formations: Sensitivity on model selection and material properties, Journal of Hydrology 531 (2015) 149-160.

[6] G. Taylor, Dispersion of soluble matter in solvent flowing slowly through a tube, Proceedings of the Royal Society of London. Series A, 219 (1953) 186-203 186-203.

[7] R. Aris, On the dispersion of solute in a fluid flowing through a tube, Proceedings of the Royal Society of London. Series A, 235 (1956) 67-77.

[8] H. Brenner, Dispersion resulting from flow through spatially periodic porous media, Philosophical Transactions of the Royal Society of London. Series A 297 (1980) 81-133. 
[9] O. Plumb, S. Whitaker, Dispersion in heterogeneous porous media: 1. local volume averaging and large-scale averaging, Water Resources Research 24 (1988) 913-926.

[10] U. Hornung, Homogenization and Porous Media, Springer, 1997.

[11] M. Shapiro, H. Brenner, Dispersion of a chemically reactive solute in a spatially periodic model of a porous medium, Chemical Engineering Science 43 (3) (1988) 551-571.

[12] B. Dykaar, P. Kitanidis, Macrotransport of a biologically reacting solute through porous media, Water Resources Research 32 (1996) 307-329.

[13] D. Bolster, F. Valdes-Parada, T. L. Borgne, M. Dentz, J. Carrera, Mixing in confined stratified aquifers, Journal of Contaminant Hydrology 120-121 (2011) 198-212.

[14] M. Levesque, O. Bénichou, R. Voituriez, B. Rotenberg, Taylor dispersion with adsorption and desorption, Physical Review E 86 (3) (2012) 036316.

[15] L. Zhang, M. A. Hesse, M. Wang, Transient solute transport with sorption in poiseuille flow, Journal of Fluid Mechanics 828 (2017) 733-752. doi:10.1017/jfm.2017.546.

[16] I. Battiato, D. M. Tartakovsky, A. M. Tartakovsky, T. Scheibe, On breakdown of macroscopic models of mixing-controlled heterogeneous reactions in porous media, Advances in Water Resources 32 (11) (2009) 1664-1673. 
[17] I. Battiato, D. Tartakovsky, Applicability regimes for macroscopic models of reactive transport in porous media, Journal of Contaminant Hydrology 120 (2011) 18-26.

[18] K. Rehfeldt, J. Boggs, L. Gelhar, Field study of dispersion in a heterogeneous aquifer. 3: Geostatistical analysis of hydraulic conductivity, Water Resources Research 28 (1992) 3309-3324.

[19] C. Harvey, S. M. Gorelick, Rate-limited mass transfer or macrodispersion: Which dominates plume evolution at the macrodispersion experiment (made) site?, Water Resources Research 36 (3) (2000) 637-650.

[20] A. Cortis, B. Berkowitz, Anomalous transport in "classical" soil and sand columns, Soil Science Society of America Journal 68 (5) (2004) $1539-1548$.

[21] M. Dentz, A. Cortis, H. Scher, B. Berkowitz, Time behavior of solute transport in heterogeneous media: transition from anomalous to normal transport, Advances in Water Resources 27 (2) (2004) 155-173.

[22] Y. Zhang, D. A. Benson, B. Baeumer, Predicting the tails of breakthrough curves in regional-scale alluvial systems, Groundwater 45 (4) (2007) 473-484.

[23] M. C. Richmond, W. A. Perkins, T. D. Scheibe, A. Lambert, B. D. Wood, Flow and axial dispersion in a sinusoidal-walled tube: Effects of inertial and unsteady flows, Advances in Water Resources.

[24] M. L. Porter, F. J. Valdés-Parada, B. D. Wood, Comparison of theory 
and experiments for dispersion in homogeneous porous media, Advances in Water Resources 33 (9) (2010) 1043-1052.

[25] G. Porta, G. Ceriotti, J.-F. Thovert, Comparative assessment of continuum-scale models of bimolecular reactive transport in porous media under pre-asymptotic conditions, Journal of contaminant hydrology 185 (2016) 1-13.

[26] Y. Davit, B. D. Wood, G. Debenest, M. Quintard, Correspondence between one-and two-equation models for solute transport in two-region heterogeneous porous media, Transport in porous media 95 (1) (2012) 213-238.

[27] T. L. Borgne, M. Dentz, J. Carrera, Lagrangian statistical model for transport in highly heterogeneous velocity fields, Physical Review Letters 101 (2008) 090601.

[28] N. L. Sund, D. Bolster, D. A. Benson, Testing the limits of the spatial markov model for upscaling transport: The role of nonmonotonic effective velocity autocorrelations, Physical Review E 94 (4) (2016) 043107.

[29] B. Berkowitz, A. Cortis, M. Dentz, H. Scher, Modeling non-Fickian transport in geological formations as a continuous time random walk, Reviews of Geophysics 44 (2).

[30] D. Bolster, Y. Méheust, T. Le Borgne, J. Bouquain, P. Davy, Modeling preasymptotic transport in flows with significant inertial and trapping effects-the importance of velocity correlations and a spatial Markov model, Advances in Water Resources 70 (2014) 89-103. 
[31] T. L. Borgne, M. Dentz, J. Carrera, Spatial Markov processes for modeling Lagrangian particle dynamics in heterogeneous porous media, Physical Review E 78 (2008) 026308.

[32] P. K. Kang, M. Dentz, T. Le Borgne, R. Juanes, Spatial Markov model of anomalous transport through random lattice networks, Physical Review Letters 107 (18) (2011) 180602.

[33] T. Le Borgne, D. Bolster, M. Dentz, P. Anna, A. Tartakovsky, Effective pore-scale dispersion upscaling with a correlated continuous time random walk approach, Water Resources Research 47 (12).

[34] P. De Anna, T. Le Borgne, M. Dentz, A. M. Tartakovsky, D. Bolster, P. Davy, Flow intermittency, dispersion, and correlated continuous time random walks in porous media, Physical Review Letters 110 (18) (2013) 184502.

[35] P. K. Kang, P. Anna, J. P. Nunes, B. Bijeljic, M. J. Blunt, R. Juanes, Pore-scale intermittent velocity structure underpinning anomalous transport through 3-d porous media, Geophysical Research Letters 41 (17) (2014) 6184-6190.

[36] N. Sund, D. Bolster, S. Mattis, C. Dawson, Pre-asymptotic transport upscaling in inertial and unsteady flows through porous media, Transport in Porous Media 109 (2) (2015) 411-432.

[37] P. K. Kang, T. Le Borgne, M. Dentz, O. Bour, R. Juanes, Impact of velocity correlation and distribution on transport in fractured media: Field evidence and theoretical model, Water Resources Research. 
[38] N. L. Sund, D. Bolster, C. Dawson, Upscaling transport of a reacting solute through a peridocially converging-diverging channel at preasymptotic times, Journal of contaminant hydrology 182 (2015) 1-15.

[39] N. Sund, G. Porta, D. Bolster, R. Parashar, A lagrangian transport eulerian reaction spatial (laters) markov model for prediction of effective bimolecular reactive transport, Water Resources Research 53 (11) (2017) 9040-9058.

[40] N. L. Sund, G. M. Porta, D. Bolster, Upscaling of dilution and mixing using a trajectory based spatial markov random walk model in a periodic flow domain, Advances in Water Resources 103 (2017) 76-85.

[41] T. Bahar, F. Golfier, C. Oltéan, E. Lefevre, C. Lorgeoux, Comparison of theory and experiment for napl dissolution in porous media, Journal of Contaminant Hydrology 211 (2018) 49-64. doi:10.1016/j.jconhyd.2018.03.004.

[42] G. Boccardo, E. Crevacore, R. Sethi, M. Icardi, A robust upscaling of the effective particle deposition rate in porous media, Journal of Contaminant Hydrology 212 (2018) 3-13. doi:10.1016/j.jconhyd.2017.09.002.

[43] P. K. Kang, S. Brown, R. Juanes, Emergence of anomalous transport in stressed rough fractures, Earth and Planetary Science Letters 454 (2016) 46-54.

[44] T. Sherman, A. Fakhari, S. Miller, K. Singha, D. Bolster, Parameterizing the spatial markov model from breakthrough curve data alone, Water Resources Research 53 (12) (2017) 10888-10898. 
[45] T. Sherman, A. Foster, D. Bolster, K. Singha, Predicting downstream concentration histories from upstream data in column experiments, Water Resources Research ?? (2018) Under Review.

[46] B. Rathi, H. Neidhardt, M. Berg, A. Siade, H. Prommer, Processes governing arsenic retardation on pleistocene sediments: Adsorption experiments and model-based analysis, Water Resources Research 53 (5) (2017) 4344-4360. doi:10.1002/2017WR020551.

[47] O. Iliev, Z. Lakdawala, K. Neßler, T. Prill, Y. Vutov, Y. Yang, J. Yao, On the pore-scale modeling and simulation of reactive transport in $3 \mathrm{~d}$ geometries, Mathematical Modelling and Analysis 22 (5) (2017) 671694. doi:10.3846/13926292.2017.1356759.

[48] C. da Luz, S. de Arruda Guelli Ulson de Souza, A. Ulson de Souza, A. Dervanoski, A. de Oliveira Samel Moraes, B. Wood, A multiscale model for carbon adsorption of btx compounds: Comparison of volume averaging theory and experimental measurements, Chemical Engineering Science 184 (2018) 285-308. doi:10.1016/j.ces.2018.02.047.

[49] N. Seetha, M. Mohan Kumar, S. Majid Hassanizadeh, A. Raoof, Virussized colloid transport in a single pore: Model development and sensitivity analysis, Journal of Contaminant Hydrology 164 (2014) 163-180. doi:10.1016/j.jconhyd.2014.05.010.

[50] Y. Edery, A. Guadagnini, H. Scher, B. Berkowitz, Origins of anomalous transport in heterogeneous media: Structural and dynamic controls, Water Resources Research 50 (2) (2014) 1490-1505. 
[51] K. Chaudhary, M. Cardenas, W. Deng, P. Bennett, Pore geometry effects on intrapore viscous to inertial flows and on effective hydraulic parameters, Water Resources Research 49 (2013) 1149-1162.

[52] K. Chaudhary, M. B. Cardenas, W. Deng, P. C. Bennett, The role of eddies inside pores in the transition from Darcy to Forchheimer flows, Geophysical Research Letters 38 (24).

[53] J. Bouquain, Y. Meheust, D. Bolster, P. Davy, The impact of inertial effects on solute dispersion in a channel with periodically varying aperture, Physics of Fluids 24 (2012) 083602.

[54] M. B. Cardenas, Direct simulation of pore level Fickian dispersion scale for transport through dense cubic packed spheres with vortices, Geochem., Geophs., Geosyst. 10 (2009) Q12014.

[55] D. Bolster, T. L. Borgne, M. Dentz, Solute dispersion in channels with periodically varying apertures, Physics of Fluids 21 (2009) 056601.

[56] M. B. Cardenas, Three-dimensional vortices in single pores and their effects on transport, Geophysical Research Letters 35 (18).

[57] T. LeBorgne, D. Bolster, M. Dentz, P. de Anna, A. Tartakovsky, Effective pore-scale dispersion upscaling with a correlated CTRW approach, Water Resources Research 47 (2012) W12538.

[58] P. Kitanidis, B. Dykaar, Stokes flow in a slowly varying two-dimensional periodic pore, Transport in Porous Media 26 (1997) 89-98. 
[59] J. Cao, P. Kitanidis, Adaptive finite element simulation of stokes flow in porous media, Advances in water resources 22 (1) (1998) 17-31.

[60] M. Khan, Non-equilibrium theory of capillary columns and the effect of interfacial resistance on column efficiency, Gas Chromatography (1962) $3-17$.

[61] T. Ptak, G. Teutsch, Forced and natural gradient tracer tests in a highly heterogeneous porous aquifer: instrumentation and measurements, Journal of Hydrology 159 (1-4) (1994) 79-104.

[62] V. L. Morales, M. Dentz, M. Willmann, M. Holzner, Stochastic dynamics of intermittent pore-scale particle motion in three-dimensional porous media: Experiments and theory, Geophysical Research Letters 44 (18) (2017) 9361-9371.

[63] M. Dentz, P. K. Kang, A. Comolli, T. Le Borgne, D. R. Lester, Continuous time random walks for the evolution of lagrangian velocities, Physical Review Fluids 1 (7) (2016) 074004.

[64] A. Comolli, M. Dentz, Anomalous dispersion in correlated porous media: a coupled continuous time random walk approach, The European Physical Journal B 90 (9) (2017) 166.

[65] P. K. Kang, M. Dentz, T. Le Borgne, S. Lee, R. Juanes, Anomalous transport in disordered fracture networks: spatial markov model for dispersion with variable injection modes, Advances in Water Resources 106 (2017) 80-94. 
766

767

768

769

770

771

772

773

774

775

776

777

778

[66] H. Risken, Fokker-Planck Equation, Springer, 1984.

[67] G. Boccardo, I. M. Sokolov, A. Paster, An improved scheme for a robin boundary condition in discrete-time random walk algorithms, Journal of Computational Physics 374 (2018) 1152-1165.

[68] E. Wright, N. Sund, D. Richter, G. Porta, D. Bolster, Upscaling mixing in highly heterogeneous porous media via a spatial markov model, Water 11 (1) (2019) 53.

[69] S. Most, Analysis and simulation of anomalous transport in porous media, Ph.D. thesis, University of Stuttgart (2019).

[70] A. Massoudieh, M. Dentz, J. Alikhani, A spatial m arkov model for the evolution of the joint distribution of groundwater age, arrival time, and velocity in heterogeneous media, Water Resources Research 53 (7) (2017) 5495-5515. 